\title{
Therapeutic challenges of psoriasis in the HIV-infected patient: A case report
}

\author{
MANUELA ARBUNE ${ }^{1,2^{*}}$, ANCA-ADRIANA ARBUNE ${ }^{3}$, ELENA NICULET $^{4,5}$, \\ LUCRETIA ANGHEL $^{1 *}$, SILVIA FOTEA ${ }^{1^{*}}$ and ALIN LAURENTIU TATU ${ }^{1,6,7}$

\footnotetext{
${ }^{1}$ Department of Clinical Medicine, Faculty of Medicine and Pharmacy, 'Dunărea de Jos' University, 800010 Galati;

${ }^{2}$ Department of Infectious Diseases, 'Sf. Cuvioasa Parascheva' Clinical Hospital of Infectious Diseases, 800179 Galati;

${ }^{3}$ Department of Neurology, 'Fundeni' Clinical Institute, 022328 Bucharest; ${ }^{4}$ Department of Morphological and

Functional Sciences, Faculty of Medicine and Pharmacy, 'Dunărea de Jos’ University, 800010 Galați;

${ }^{5}$ Department of Pathology, 'Sfantul Apostol Andrei' Emergency Clinical Hospital, 800578 Galati;

${ }^{6}$ Research Center in the Field of Medical and Pharmaceutical Sciences, ReFORM-UDJ, 800010 Galati;

${ }^{7}$ Department of Dermatology, 'Sf. Cuvioasa Parascheva' Clinical Hospital of Infectious Diseases, 800179 Galati, Romania
}

Received August 13, 2021; Accepted September 14, 2021

DOI: $10.3892 /$ etm.2021.11098

\begin{abstract}
Psoriasis can be paradoxically associated with human immunodeficiency virus (HIV) infection, having a prevalence similar to the general population but with a more severe evolution. In the genetically predisposed patients with the $\mathrm{CW}^{*} 0602$ haplotype, HIV infection can be a triggering factor and a first sign of infection, and lesions can spontaneously remit with immune reconstruction after antiretroviral therapy. Our patient is a 34 year-old male with recent HIV infection, in spite of being for over 10 years the partner of an HIV-positive patient with whom the patient has two HIV-positive children. The patient was diagnosed with psoriasis 7 years ago and was treated topically. The physical
\end{abstract}

Correspondence to: Dr Anca-Adriana Arbune, Department of Neurology, 'Fundeni' Clinical Institute, 258 Fundeni Street, 022328 Bucharest, Romania

E-mail: arbunemanuela@yahoo.com

Dr Elena Niculet, Department of Morphological and Functional Sciences, Faculty of Medicine and Pharmacy, 'Dunărea de Jos' University, 35 Alexandru Ioan Cuza Street, 800010 Galati, Romania E-mail: helena_badiu@yahoo.com

*Contributed equally

Abbreviations: HIV, human immunodeficiency virus; AIDS, acquired immunodeficiency syndrome; IL2, interleukin-2; INF- $\gamma$, interferon- $\gamma$; TNF- $\alpha$, tumor necrosis factor- $\alpha$; UV, ultraviolet; MTX, methotrexate; BMI, body mass index; RNA-HIV, ribonucleic acid HIV; HBV, hepatitis B virus; HCV, hepatitis C virus; CMV, cytomegalovirus; PASI, Psoriasis Area and Severity Index; ALT, alanine aminotransferase; HAART, highly active antiretroviral therapy

Key words: psoriasis, HIV, methothrexate, triumeq, biologics examination at HIV diagnosis was overall favorable, with skin findings compatible with disseminated vulgar psoriasis. Following antiretroviral treatment with Triumeq the patient had a favorable viral response, with complete viral suppression after 12 weeks, but the pre-existent psoriasis lesions worsened. Methotrexate (MTX) treatment followed for 12 weeks, with partial improvement of psoriatic dermatitis. This medication was continued for 1 year, but the lesions reappeared, possibly due to treatment resistance. MTX treatment for psoriasis in the HIV-infected patient was beneficial, but limited to one year, leaving biologics as possible treatment following therapy under strict monitoring for adverse effects, T-lymphocyte $\mathrm{CD}^{+}$and viral levels.

\section{Introduction}

Psoriasis is an autoimmune disease with chronic course, having relapse and remission periods and a variable European country prevalence from 1.3 to $11.4 \%(1-3)$.

Psoriasis may be associated with human immunodeficiency virus (HIV) infection, but the prevalence of the viral infection does not differ significantly from that of the general population. Although it is not a characteristic presentation of the acquired immunodeficiency syndrome (AIDS), the evolution of psoriasis can be more severe in patients with HIV (4-6).

HIV infection can be a triggering factor for psoriasis development and a first sign of infection in genetically predisposed patients having the $\mathrm{CW}^{*} 0602$ haplotype (7). In these patients, psoriasis lesions could improve once immune reconstruction has taken place after antiretroviral therapy, reaching even spontaneous remission. That being said, some guttate and vulgar forms of psoriasis can worsen in the advanced stages of AIDS (4).

The pathogenesis of associating psoriasis to HIV-infected patients is paradoxical and is explained by the imbalance between immune cellular components. $\mathrm{T}$ lymphocytes have the main purpose of mediating the excessive inflammatory 
Table I. Biologic outcome in an HIV-positive patient suffering from psoriasis, under MTX treatment.

\begin{tabular}{|c|c|c|c|c|}
\hline Timeline & February 2019 & May 2019 & September 2019 & September 2020 \\
\hline $\mathrm{Hb}(\mathrm{g} / \mathrm{dl})$ & 15.2 & 15.4 & 14.5 & 15.5 \\
\hline WBC $\left(\right.$ cells $\left./ \mathrm{mm}^{3}\right)$ & 8,110 & 6,310 & 9,600 & 11,000 \\
\hline ALT (UI/l) & 24.2 & 31 & 24.6 & 96.3 \\
\hline AST (UI/l) & 22 & 28.2 & 26.4 & 66.8 \\
\hline Creatinine (mg/dl) & 0.82 & 0.79 & 0.74 & 0.75 \\
\hline T-lymphocyte CD4 (cells $\left./ \mathrm{mm}^{3}\right)$ & 466 & 581 & 450 & 500 \\
\hline ARN-HIV (copies/ml) & 146,000 & Undetectable & Undetectable & Undetectable \\
\hline TRIUMEQ & $\sqrt{ }$ & $\sqrt{ }$ & $\sqrt{ }$ & $\sqrt{ }$ \\
\hline MTX & & $\sqrt{ }$ & $\sqrt{ }$ & $\sqrt{ }$ \\
\hline PASI (\%) & 21.2 & 19 & 17 & 16.2 \\
\hline
\end{tabular}

HIV, human immunodeficiency virus; ALT, alanine aminotransferase; AST, aspartate aminotransferase; MTX, methotrexate; PASI, Psoriasis Area and Severity Index. $\sqrt{ }$ indicates that the patient was receiving treatment with the relevant agent during this time period.

response that takes place in psoriasis, while HIV infection is characterized by T-lymphocyte depletion (a state which renders the body susceptible to various opportunistic infections) (8). The cytokine profile of the two diseases is also different, psoriasis having a Th1 pattern [interleukin-2 (IL2), interferon- $\gamma(\mathrm{INF}-\gamma)$, tumor necrosis factor- $\alpha$ (TNF- $\alpha)$, while HIV infection has a Th2 pattern (IL-2, IL-5, IL-10) (9-11).

Psoriasis biological therapies which target lymphocyte Th1 contribute to the severity of HIV infection progression, while antiretroviral therapy has the capacity to improve psoriatic lesions. However, some case reports have revealed that antiretroviral therapy initiation in severe immune-suppressed patients, especially when T-lymphocyte $\mathrm{CD}^{+}$levels are below 50 cells $/ \mathrm{mm}^{3}$, could be associated with a temporary exacerbation of psoriasis, within the immune reconstruction inflammatory syndrome (12).

Available therapies for HIV-associated psoriasis are classified into 3 categories, producing results that vary from complete remission to lack of any response. Local therapy uses tar products, emollients, salicylic acid, corticosteroids and retinoids. Ultraviolet (UV) phototherapy is controversial. Systemic therapy is based on acitretin, methotrexate (MTX) and cyclosporine, anti-TNF- $\alpha$ biologic therapy. MTX is rarely used in HIV patients due to its immune-modulating effect, renal toxicity or the folate metabolism (13-16).

Previous studies have revealed that the purpose of Th17 lymphocytes in natural and adaptive immunity in skin and mucous membranes, is their involvement in the pathogenesis of autoimmune, neoplastic and infectious processes at this level. Th17 lymphocytes are a Th-lymphocyte subset, which produce cytokines that act on epithelial cells, thus becoming a target for research in patients with both psoriasis and HIV infection, in the hopes of finding new therapies (13-15).

\section{Case report}

The case of a 34-year-old male, unqualified construction worker from a rural environment with a primary school education level, is presented, who addressed the 'Sf. Cuvioasa Parascheva' Clinical Hospital of Infectious Diseases (Galati,
Romania) for the evaluation, monitoring and treatment of his recent HIV infection. For over 10 years the patient has been the partner of a positive HIV patient, with whom the patient has two seropositive HIV children, systematically breaching the safe sex measures. His last HIV testing, two years ago, was negative. The patient was a chronic smoker (23 pack-years) and alcohol consumer (4 units/day). A total of 18 years ago, the patient suffered multiple trauma involving cranial-cerebral injuries and coma, being hospitalized for over a month. A total of 7 years prior to the HIV diagnosis (when the patient was 28), the patient received the clinical diagnosis of psoriasis and followed various topical treatments. The family history is negative for psoriasis and the patient suffers from low self-image with poor quality of life due to the appearance and discomfort of the psoriatic lesions.

Upon initial clinical examination, when the patient was diagnosed as being HIV-positive, his general health status was favorable, the patient was afebrile with a body mass index (BMI) of $21,2 \mathrm{~kg} / \mathrm{m}^{2}$ and blood pressure of $150 / 100 \mathrm{mmHg}$, with rhythmic heartbeats and no additional heart sounds; the lung sounds were normal, as was the neurologic examination. Mucosal examination revealed candidiasis lesions. Onychomycosis and squamous scalp lesions were also identified, and the skin examination revealed disseminated vulgar psoriasis lesions. Laboratory data revealed an $\mathrm{CD}^{+}$ T-lymphocyte number of 466 cells $/ \mathrm{mm}^{3}$, a ribonucleic acid (RNA)-HIV viral load of 146,000 copies/ml, with negative serologic examination for hepatitis B virus (HBV), hepatitis $\mathrm{C}$ virus (HCV), cytomegalovirus (CMV), toxoplasma and syphilis. The remaining hematological and biochemical investigations were in the normal ranges, while no significant lesions were found on the thoracic X-ray.

The patient received antiretroviral treatment with Triumeq (dolutegravir + lamivudine + abacavir), registering a favorable viral response with complete viral suppression after 12 weeks. The treatment was well tolerated, but the pre-existent psoriatic lesions treated with topical medication became more extended and accentuated the inflammatory response with a Psoriasis Area and Severity Index (PASI) score of 21,2. The patient then received systemic treatment with MTX as $15 \mathrm{mg} /$ week, 


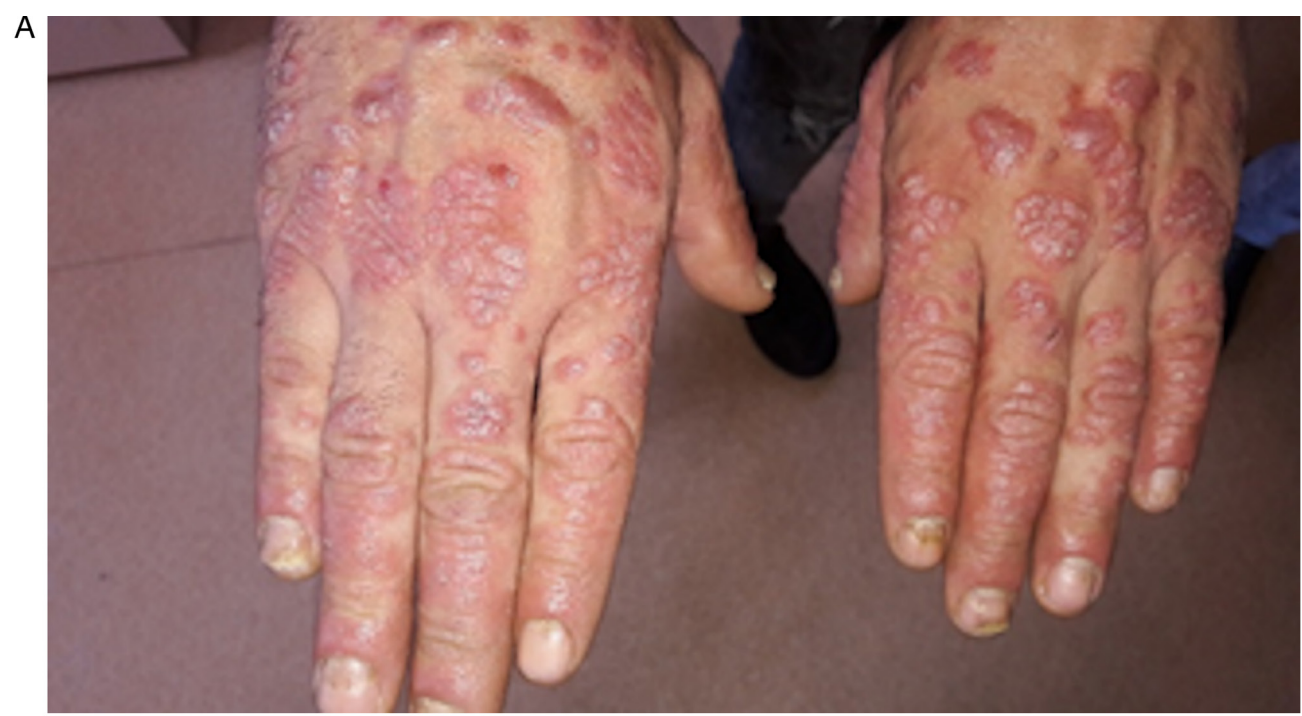

B
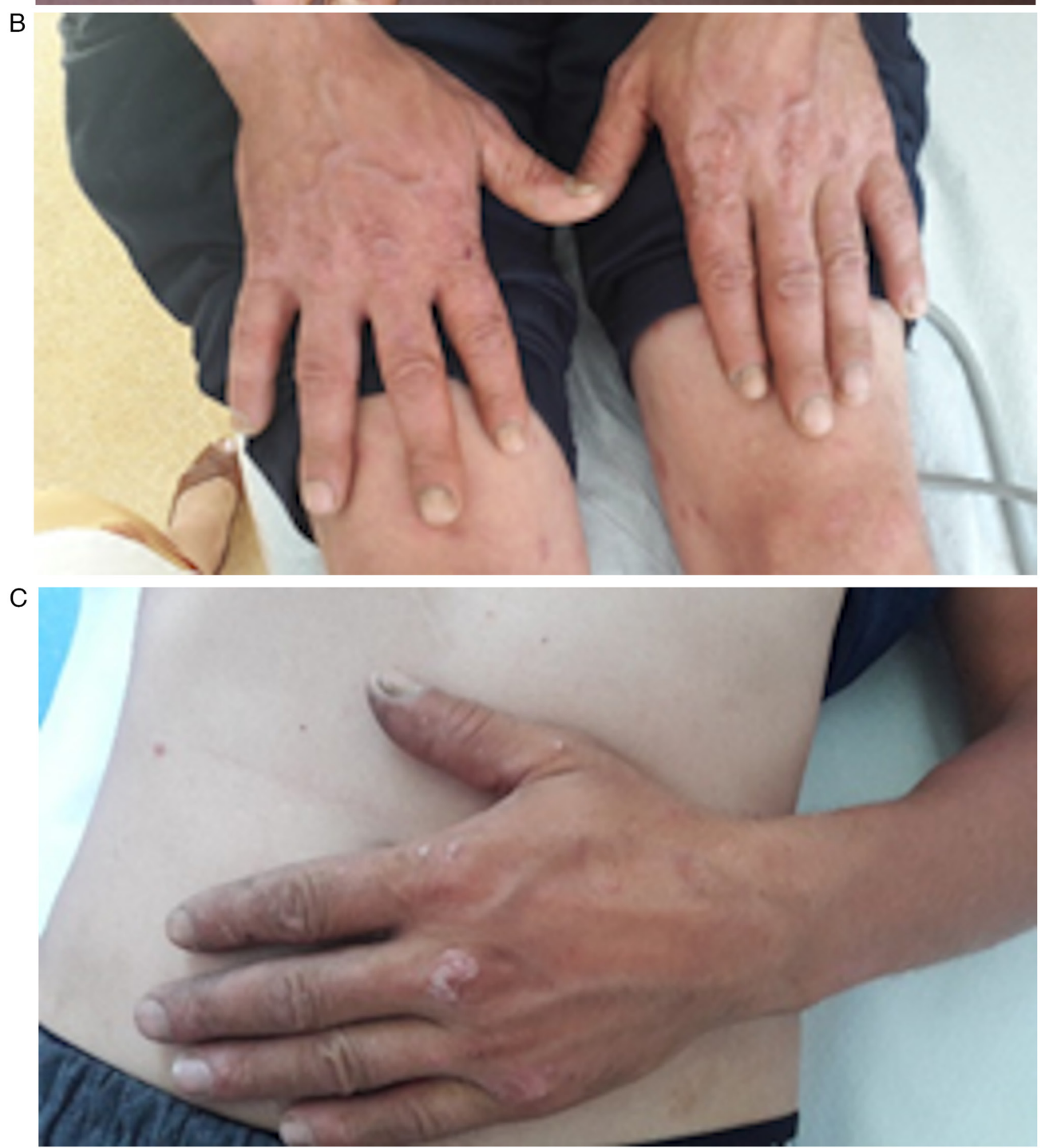

Figure 1. Clinical outcome of psoriasis under MTX treatment in an HIV-positive patient. (A) At the beginning of treatment. (B) After 4 months of treatment. (C) After 1 year of treatment.

for 12 weeks, with major improvement of lesions and partial remission. Under strict biologic monitoring, MTX treatment was continued with the same dosage for a year, but the lesions reappeared, coinciding with a three-fold increase in alanine aminotransferase (ALT) levels. The laboratory changes during MTX treatment (from May 2019 to September 2020) are highlighted in Table I, along with the evolution of hand skin lesions in Fig. 1. 


\section{Discussion}

MTX is an immune suppressive drug frequently used in autoimmune and inflammatory dermatological diseases $(17,18)$.

Current treatment guidelines recommend MTX as first-line systemic treatment in any form of psoriasis which cannot be controlled by topical therapy, which significantly affects the quality of life of the patient and fulfills one of the following conditions: is extensive (PASI $>10 \%$ ); is localized, but is associated with significant functional deterioration; or phototherapy cannot be used, is inefficient or has led to a fast relapse. Concerning severity scores after MTX use for 12-24 weeks, randomized prospective studies conducted on large cohorts of patients suffering from psoriasis, proved to have similar efficacy as biological therapies with adalimumab, briakinumab, infliximab, but also as with cyclosporine (17).

MTX use in HIV patients is associated with a high risk for opportunistic infections. Medical literature data has reported a case of toxic encephalopathy which improved after cessation of MTX (19). In another study, 2 out of the 4 patients treated with MTX suffered from pneumocystis pneumonia, although they received prophylaxis for opportunistic infections prior to starting treatment (19). Having associated infections with HBV, $\mathrm{HCV}$ or latent tuberculosis are absolute contraindications for MTX use $(19,20)$, but in our case the serologic markers of the patients for these infections were negative.

The medical literature has few data concerning the efficacy of systemic therapies in HIV-positive patients suffering from psoriasis, most recommendations exerting caution in their use. However, the American Academy of Dermatology collected data on the use of TNF- $\alpha$ inhibitors (etanercept, adalimumab and infliximab) in HIV-positive patients suffering from psoriatic arthritis who did not respond to the combination treatment with MTX and cyclosporine, adherent to the antiretroviral therapy with encouraging results $(20,21)$. Ustekinumab is a p40-IL 12/23 inhibitor that was used for refractory forms of HIV-associated psoriasis, without registering any adverse effects in the first months of treatment $(20,22)$.

Concerning certolizumab, a TNF- $\alpha$ inhibitor and an approved biologic drug for plaque psoriasis and for psoriatic arthritis, this is an agent that could be used as systemic treatment in HIV-positive patients suffering from psoriasis, having an undetectable viral load and under highly active antiretroviral therapy (HAART) $(23,24)$. Risankizumab and guselkumab are both biologicals approved for targeting the p19 subunit of IL-23 (IL23/p19 inhibitors), used in moderate to severe plaque psoriasis. The advantage of using these two drugs would be an improved safety profile (as compared with TNF- $\alpha$ inhibitors) $(23,25)$. Although guselkumab is reported to be safe and effective in HIV-positive patients suffering from psoriasis, on condition of close monitoring, there remains insufficient data for guselkumab and risankizumab use in these particular patients. These three agents could have positive effects on the T-lymphocyte $\mathrm{CD} 4^{+}$counts and on the viral load $(17,23,25)$.

The case features were as follows: i) In spite of repeated unprotected sexual contact, the patient suffering from psoriasis was not infected with HIV for a long time, suggesting a strong immune response with a particular genetic determinism; ii) psoriasis was diagnosed prior to HIV and its onset was at a young age, corresponding most probably to type I psoriasis; iii) the genetic profile $\mathrm{CW}^{*} 0602$ remained uninvestigated, having no family links to other cases of psoriasis; iv) the evolution of psoriasis could be favored by external factors including alcohol, smoking, excessive sun exposure, hygiene deficit, which were not corrected during therapy; v) antiretroviral treatment was followed by immune recovery and complete viral suppression; vi) psoriasis exacerbation after the first weeks of efficient antiviral treatment could correspond to a rare paradoxical inflammatory syndrome of immune reconstruction; vii) topical therapy resistance and controversies regarding the efficacy and safety of phototherapy in the HIV-positive patient justified the decision of using MTX as first-line systemic treatment; viii) MTX use in the patient with antiviral therapy-controlled HIV infection, was well tolerated and efficient, with a partial remission of psoriasis lesions in the first 12 weeks; and ix) re-emergence of lesions after one year of continuation of MTX could be explained by the development of treatment resistance, making it necessary to apply or associate other therapeutic options.

In conclusion, in the context of HIV infection, progression of psoriasis may be an expression of specific viral pathogenic immune deficiency, but also of inflammatory processes which accompany immune reconstruction after antiretroviral therapy. MTX use for severe psoriasis treatment was an efficient and safe solution for dermatological lesion control in the HIV-infected patient, but the benefits were limited to only one year. Biological therapies are the future options for psoriasis treatment after MTX failure, but they require rigorous monitoring for adverse effects, $\mathrm{CD} 4^{+}$ lymphocytes and viral levels.

\section{Acknowledgements}

Not applicable.

\section{Funding}

The present study was supported by 'Dunarea de Jos' University of Galati.

\section{Availability of data and materials}

The datasets used and/or analyzed during the current study are available from the corresponding author on reasonable request.

\section{Authors' contributions}

MA and AAA confirm the authenticity of all the raw data. MA, AAA, EN, SF, LA and ALT were major contributors in writing the manuscript, were involved in all the stages of the study, contributed to the conception and design of the work, as well as revising it and have also contributed in analyzing the data for the study. MA, AAA, EN, SF, LA and ALT revised it for important intellectual content. MA, AAA, EN, SF, LA and ALT approved the final version to be published. All authors read and approved the final manuscript and agree to be accountable for all aspects of the work in ensuring that questions related to the accuracy or integrity of any part of the work are appropriately investigated and resolved. 


\section{Ethics approval and consent to participate}

Ethics approval was granted by the Ethics Committee of the 'Sf. Cuvioasa Parascheva' Clinical Hospital of Infectious Diseases (Galati, Romania), with the decision no. 31 from 16.03.2021. The patient also provided written informed consent for participation in the study.

\section{Patient consent for publication}

The patient provided written informed consent for the publication of any associated data and accompanying figure.

\section{Competing interests}

The authors declare that they have no competing interests.

\section{References}

1. Michalek IM, Loring B and John SM: A systematic review of worldwide epidemiology of psoriasis. J Eur Acad Dermatol Venereol 31: 205-212, 2017

2. Batani A, Brănisteanu DE, Ilie MA, Boda D, Ianosi S, Ianosi G and Caruntu C: Assessment of dermal papillary and microvascular parameters in psoriasis vulgaris using in vivo reflectance confocal microscopy. Exp Ther Med 15: 1241-1246, 2018.

3. Caruntu C, Boda D, Căruntu A, Rotaru M, Baderca F and Zurac S: In vivo imaging techniques for psoriatic lesions. Rom J Morphol Embryol 55 (3 Suppl): S1191-S1196, 2014.

4. Dlova NC and Mosam A: Inflammatory noninfectious dermatoses of HIV. Dermatol Clin 24: 439-448, VI, 2006.

5. Mamkin I, Mamkin A and Ramanan SV: HIV-associated psoriasis. Lancet Infect Dis 7: 496, 2007.

6. Jordaan HF: Common skin and mucosal disorders in HIV/AIDS S Afr Fam Pract 50: 14-23, 2008.

7. Cedeno-Laurent F, Gómez-Flores M, Mendez N, Ancer-Rodríguez J, Bryant JL, Gaspari AA and Trujillo JR: New insights into HIV-1-primary skin disorders. J Int AIDS Soc 14: 5,2011 .

8. Fife DJ, Waller JM, Jeffes EW and Koo JY: Unraveling the paradoxes of HIV-associated psoriasis: A review of T-cell subsets and cytokine profiles. Dermatol Online J 13: 4, 2007.

9. Friedrich M, Krammig S, Henze M, Döcke WD, Sterry W and Asadullah K: Flow cytometric characterization of lesional $\mathrm{T}$ cells in psoriasis: Intracellular cytokine and surface antigen expression indicates an activated, memory/effector type $1 \mathrm{immu}$ nophenotype. Arch Dermatol Res 292: 519-521, 2000.

10. Mallon E and Bunker CB: HIV-associated psoriasis. AIDS Patient Care STDS 14: 239-246, 2000.

11. Niculet E, Radaschin DS, Nastase F, Draganescu M, Baroiu L, Miulescu M, Arbune M and Tatu AL: Influence of phytochemicals in induced psoriasis (review). Exp Ther Med 20: 3421-3424, 2020 .
12. Cheaito MA, Khalifeh M, Jaafar B and Rizk N: Immune reconstitution inflammatory syndrome presenting as psoriasis after initiating antiretroviral therapy: A case-report. J Infect Dis Ther 6: 387, 2018.

13. Saketkoo LA and Espinoza LR: Impact of biologic agents on infectious diseases. Infect Dis Clin North Am 20: 931-961, viii, 2006.

14. Boda D, Negrei C, Nicolescu F and Badalau C: Assessment of some oxidative stress parameters in methotrexate treated psoriasis patients. Farmacia 62: 704-710, 2014.

15. Caruntu C, Boda D, Dumitrascu G, Constantin C and Neagu M: Proteomics focusing on immune markers in psoriatic arthritis. Biomark Med 9: 513-528, 2015.

16. Teh YC, Robinson S, Tan WC, Kwan Z and Tang MM: Psoriasis patients with human immunodeficiency virus infection: Data from the malaysian psoriasis registry. Malaysian J Dermatol 46: $1-10,2021$.

17. Warren RB, Weatherhead SC, Smith CH, Exton LS, Mohd Mustapa MF, Kirby B and Yesudian PD: British association of dermatologists' guidelines for the safe and effective prescribing of methotrexate for skin disease 2016. Br J Dermatol 175: 23-44, 2016.

18. Ezhilarasan D: Hepatotoxic potentials of methotrexate: Understanding the possible toxicological molecular mechanisms. Toxicology 458: 152840, 2021.

19. Kaushik SB and Lebwohl MG: Psoriasis: Which therapy for which patient: Focus on special populations and chronic infections. J Am Acad Dermatol 80: 43-53, 2019.

20. Queirós N and Torres T: HIV-associated psoriasis. Actas Dermosifiliogr (Engl Ed) 109: 303-311, 2018 (In English, Spanish).

21. Gallitano SM, McDermott L, Brar K and Lowenstein E: Use of tumor necrosis factor (TNF) inhibitors in patients with HIV/AIDS. J Am Acad Dermatol 74: 974-980, 2016.

22. Bardazzi F, Magnano M, Campanati A, Loconsole F, Carpentieri A, Potenza C, Bernardini N, Di Lernia V, Carrera C, Raone B, et al: Biologic therapies in HIV-infected patients with psoriasis: An Italian experience. Acta Derm Venereol 97: 989-990, 2017.

23. Lambert JLW, Segaert S, Ghislain PD, Hillary T, Nikkels A, Willaert F, Lambert J and Speeckaert R: Practical recommendations for systemic treatment in psoriasis in case of coexisting inflammatory, neurologic, infectious or malignant disorders (BETA-PSO: Belgian evidence-based treatment advice in psoriasis; part 2). J Eur Acad Dermatol Venereol 34: 1914-1923, 2020

24. Esposito M, Carubbi F, Giunta A, Alunno A, Giacomelli R and Fargnoli MC: Certolizumab pegol for the treatment of psoriatic arthritis and plaque psoriasis. Expert Rev Clin Immunol 16: 119-128, 2020.

25. Yang K, Oak ASW and Elewski BE: Use of IL-23 inhibitors for the treatment of plaque psoriasis and psoriatic arthritis: A comprehensive review. Am J Clin Dermatol 22: 173-192, 2021.

This work is licensed under a Creative Commons Attribution-NonCommercial-NoDerivatives 4.0 International (CC BY-NC-ND 4.0) License. 